\title{
Anatomy and white matter connections of the orbitofrontal gyrus
}

\author{
Joshua D. Burks, MD, ${ }^{1}$ Andrew K. Conner, MD, ${ }^{1}$ Phillip A. Bonney, MD, ${ }^{1}$ Chad A. Glenn, MD, ${ }^{1}$ \\ Cordell M. Baker, BS, ${ }^{1}$ Lillian B. Boettcher, BS, ${ }^{1}$ Robert G. Briggs, BS, ${ }^{1}$ Daniel L. O'Donoghue, PhD, ${ }^{2}$ \\ Dee H. Wu, PhD, ${ }^{3}$ and Michael E. Sughrue, MD ${ }^{1}$
}

Departments of ${ }^{1}$ Neurosurgery, ${ }^{2}$ Cell Biology, and ${ }^{3}$ Radiological Sciences, University of Oklahoma Health Sciences Center, Oklahoma City, Oklahoma

OBJECTIVE The orbitofrontal cortex (OFC) is understood to have a role in outcome evaluation and risk assessment and is commonly involved with infiltrative tumors. A detailed understanding of the exact location and nature of associated white matter tracts could significantly improve postoperative morbidity related to declining capacity. Through diffusion tensor imaging-based fiber tracking validated by gross anatomical dissection as ground truth, the authors have characterized these connections based on relationships to other well-known structures.

METHODS Diffusion imaging from the Human Connectome Project for 10 healthy adult controls was used for tractography analysis. The OFC was evaluated as a whole based on connectivity with other regions. All OFC tracts were mapped in both hemispheres, and a lateralization index was calculated with resultant tract volumes. Ten postmortem dissections were then performed using a modified Klingler technique to demonstrate the location of major tracts.

RESULTS The authors identified 3 major connections of the OFC: a bundle to the thalamus and anterior cingulate gyrus, passing inferior to the caudate and medial to the vertical fibers of the thalamic projections; a bundle to the brainstem, traveling lateral to the caudate and medial to the internal capsule; and radiations to the parietal and occipital lobes traveling with the inferior fronto-occipital fasciculus.

CONCLUSIONS The OFC is an important center for processing visual, spatial, and emotional information. Subtle differences in executive functioning following surgery for frontal lobe tumors may be better understood in the context of the fiber-bundle anatomy highlighted by this study.

https://thejns.org/doi/abs/10.3171/2017.3.JNS162070

KEY WORDS orbitofrontal; ventromedial; DTI; anatomy; tractography; white matter

$\mathrm{S}$ INCE Brodmann first characterized the orbitofrontal cortical region in $1909,{ }^{28}$ our knowledge of the prefrontal cortex has expanded to include its role in executive behavior and cognition. As our understanding of the prefrontal cortex has increased, it has become more apparent that its dorsolateral, ventromedial, and orbitofrontal subdivisions serve specific functions. ${ }^{3}$ For instance, it is clear that the orbitofrontal gyri are important in decision making. ${ }^{30}$ Human neuroimaging and animal lesion studies have demonstrated that this area plays a critical role in outcome evaluation and risk assessment related to anticipating reward..$^{16,18,30}$
However, an improved understanding of the role in cognition of these regions within the prefrontal cortex has yielded relatively little clinically applicable information. While speech and motor mapping have quickly become the standard for operating in speech and motor areas, it has been difficult to validate intraoperative tests for mapping regions associated with executive functioning. Adaptations of the Stroop test ${ }^{47}$ have been implemented intraoperatively to address this challenge. ${ }^{52}$ Although these cognitive tests may improve surgical outcomes, another key in preserving executive function is an understanding of the subcortical connections that host these critical networks.

ABBREVIATIONS DTI = diffusion tensor imaging; IFOF = inferior fronto-occipital fasciculus; LI = lateralization index; OFC = orbitofrontal cortex; OFG = orbitofrontal gyrus; $\mathrm{ROI}$ = region of interest; SFG = superior frontal gyrus.

SUBMITTED August 7, 2016. ACCEPTED March 23, 2017.

INCLUDE WHEN CITING Published online September 1, 2017; DOI: 10.3171/2017.3.JNS162070. 
In this paper, we show how orbitofrontal cortex (OFC) network connectivity is organized and propose adaptions of surgical techniques to save it in relevant cases. Maintaining subcortical connectivity has been shown to decrease postoperative motor deficits in patients undergoing surgery with diffusion tensor imaging (DTI)-based neuronavigation. ${ }^{55}$ Neurofunctional data has provided a basis for understanding networks involved in executive functioning, ${ }^{17,30,56}$ and these principle networks rely on various orbitofrontal areas. Despite this, the surgical anatomy of orbitofrontal connectivity has not been described in neurosurgical literature. Through DTI-based fiber tracking validated by gross anatomical dissection as ground truth, we have characterized these tracts based on key connections and anatomical relationships to other structures.

\section{Methods}

\section{Definition of Region of Interest}

There is a degree of ambiguity with respect to terminology for prefrontal areas; this is a result of multiple disciplines studying the region. From a functional standpoint, OFC is taken to refer to frontal cortical areas involved in reward processes and decision making, ${ }^{20,30}$ but from an operative perspective, distinctions based on anatomical structures are more practical. In this study, we will use gyrus-based descriptions to describe areas of connectivity. For our purposes, OFC is taken to be the anterior-most region of the cerebral cortex bounded on the posterior lateral edge by the inferior frontal gyrus (also referred to as ventrolateral cortex in functional literature ${ }^{37}$ ), posteriorly by the insula, and medially by the gyrus rectus. The gyri contained within the OFC are the anterior, posterior, medial, and lateral orbital gyri. ${ }^{42}$ This is shown in Fig. 1.

\section{Tractography}

Publicly available imaging data from the Human Connectome Project (HCP) was obtained for this study from the HCP database (http://humanconnectome.org, release Q3). Diffusion imaging from 10 healthy adult controls was analyzed. A multishell diffusion scheme was used, and the b-values were 990, 1985, and 1980 seconds $/ \mathrm{mm}^{2}$. Each bvalue was sampled in 90 directions. The in-plane resolution was $1.25 \mathrm{~mm}$. The slice thickness was $1.25 \mathrm{~mm}$. The diffusion data were reconstructed using generalized q-sampling imaging ${ }^{58}$ with a diffusion sampling length ratio of 1.25.

We performed brain registration to Montreal Neurological Institute (MNI) space, wherein imaging is warped to fit a standardized brain model for comparison between subjects. ${ }^{4}$ Tractography was performed in DSI Studio (Carnegie Mellon) using 2 predefined regions of interest (ROIs) to isolate single tracts..$^{35,58}$ Voxels within each ROI were automatically traced with randomized seeding of the voxel and/or tract with a maximum angular threshold of $45^{\circ}$. When a voxel was approached with no tract direction or a direction change greater than $45^{\circ}$, the tract was halted. Tractography was stopped after reaching a length of 450 $\mathrm{mm}$. In some instances, exclusion ROIs were placed to exclude obviously spurious tracts that were not involved in the network of interest. All tracts were dissected in both hemispheres for all regions of the OFC.
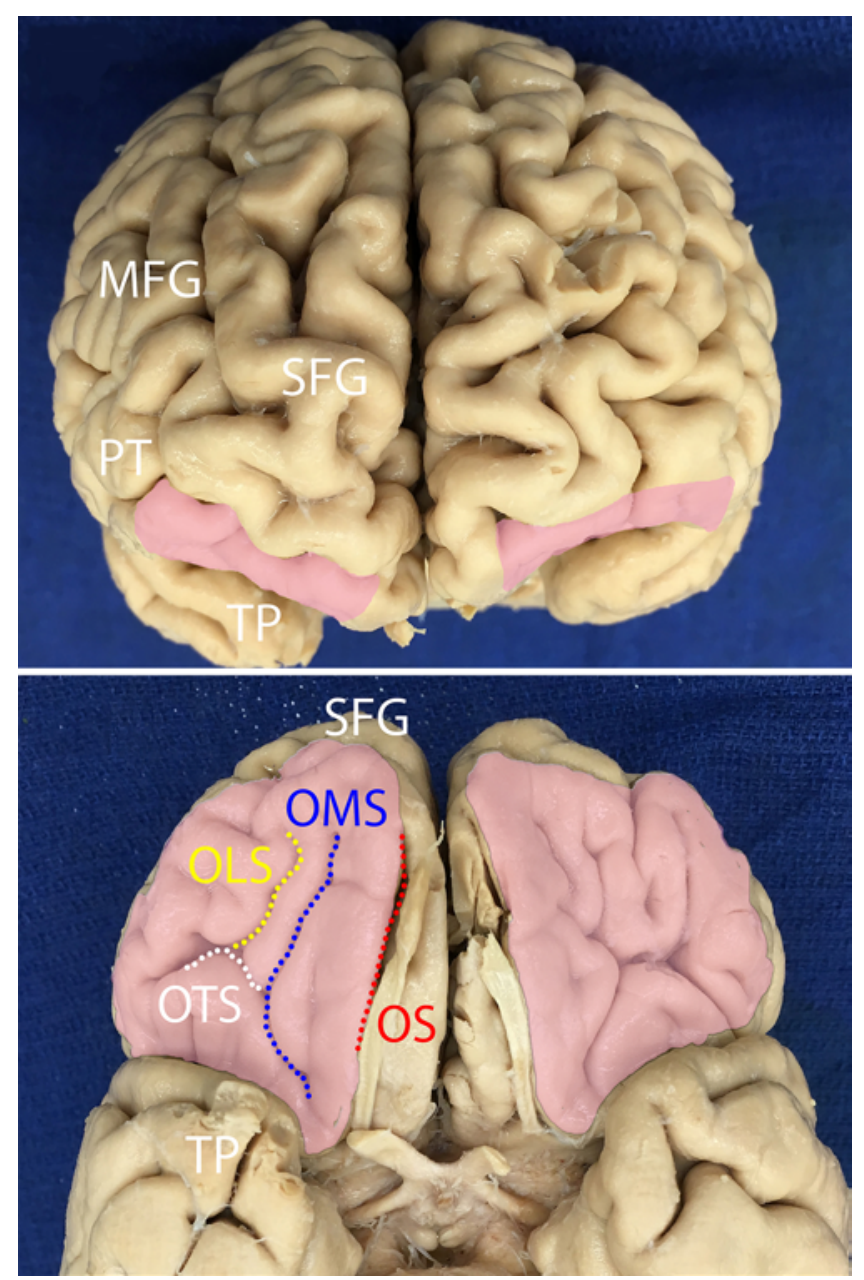

FIG. 1. Superficial anatomy of the orbitofrontal cortex (OFC). Upper: The OFC lies inferior to the superior frontal gyrus (SFG) and medial frontal gyrus (MFG) and inferomedial to the pars triangularis (PT). Lower: Basal view of the OFC. The medial orbital gyrus is lateral to the olfactory sulcus (OS) and medial to the medial orbital sulcus (OMS, blue dotted line). The lateral orbital gyrus lies lateral to the lateral orbital sulcus (OLS, yellow dotted line) and anterolateral to the transverse orbital sulcus (OTS, white dotted line). The anterior orbital gyrus is located between the OLS and OMS. Pink indicates the OFC. TP = temporal pole.

ROIs were placed manually to isolate major tracts. Tractography was performed anteriorly to posteriorly beginning with the medial gyrus, and proceeding to the anterior, posterior, and lateral gyri. All major fiber bundles reported in this study were independently identified by a minimum of 3 investigators in all subjects studied. Tractography was completed prior to cadaveric study. The lateralization index (LI) was calculated with resultant tract volumes from major identified tracts bilaterally. ${ }^{50}$ An unpaired t-test was used to assess statistical significance of any difference between these indices. A $p$ value $\leq 0.05$ was considered statistically significant.

\section{Postmortem Dissection}

The purpose of the postmortem dissections was to demonstrate the location of major tracts connecting to 
the orbitofrontal gyrus (OFG). Postmortem dissections were performed using a modified Klingler technique. ${ }^{29}$ Ten specimens were used for this study; all specimens were obtained from our institution's Willed Body Program with approval of the state's anatomical board. The cadaveric brains were fixed in $10 \%$ formalin for at least 3 months after removal from the cranium. Up until the time of dissection, the pia-arachnoid membrane was left attached.

After fixation with formalin, specimens were rinsed with water for 2 days and then frozen at $-10^{\circ} \mathrm{C}$ for 8 hours, causing white matter disruption. After thawing, dissection of the "freeze-fractured" specimen began with removal of the meninges and identification of cortical anatomy, including gyri and sulci. Relevant cortical areas were identified first. Starting superficially, they were then peeled back to reveal white matter areas of interest. Care was taken to leave cortical areas corresponding to white tracts of interest intact to preserve their relationship. Tracts were dissected with blunt instruments to avoid disrupting the natural tract anatomy. Photographs were taken at each stage of the dissection.

\section{Results}

\section{Connections to the Thalamus and Anterior Cingulate Gyrus}

Starting from the anterior portion of the medial aspect of the hemisphere, the $\mathrm{C}$-shaped cingulate sulcus is formed by the junction of the anterior cingulate gyrus and the superior frontal gyrus (SFG). A more superficial fissure divides the surface of the SFG into anterior and posterior portions. Inferiorly, the SFG is continuous with the gyrus rectus (also called the straight gyrus) along the medial edge of the hemisphere. The medial orbital gyrus rests lateral to the anterior aspect of the SFG/gyrus rectus. Fibers traversing into the superomedial portion of the thalamus pass lateral to the gyrus rectus and beneath and lateral to the caudate. Crossing fibers of the corpus callosum are superior and medial to this fiber bundle, and the vertical fibers of the thalamic projections are located laterally. Comparison with tractography with DTI is given in Fig. 2. The LI was $-0.14(\mathrm{p}=0.19)$.

\section{Brainstem Connections}

Bundles from the orbitofrontal gyri also connect with the lateral midbrain via the cerebral crus. The tract remains lateral to the caudate and medial to the internal capsule in the entirety of its anterior-posterior course. Tractography is shown for comparison in Fig. 2 and additionally demonstrates branches to the medial and lateral aspects of the OFG. More medial fibers leaving the midbrain continue anteriorly to the medial OFG, while more lateral parts of the bundle curve laterally to the lateral OFG. However, these minor branch points were not demonstrated with ex vivo dissections.

\section{Projections to Temporal, Parietal, and Occipital Lobes}

The lateral-most portion of the OFG connects to regions throughout the parietal and occipital cortex through long projections traveling with the inferior fronto-occip-
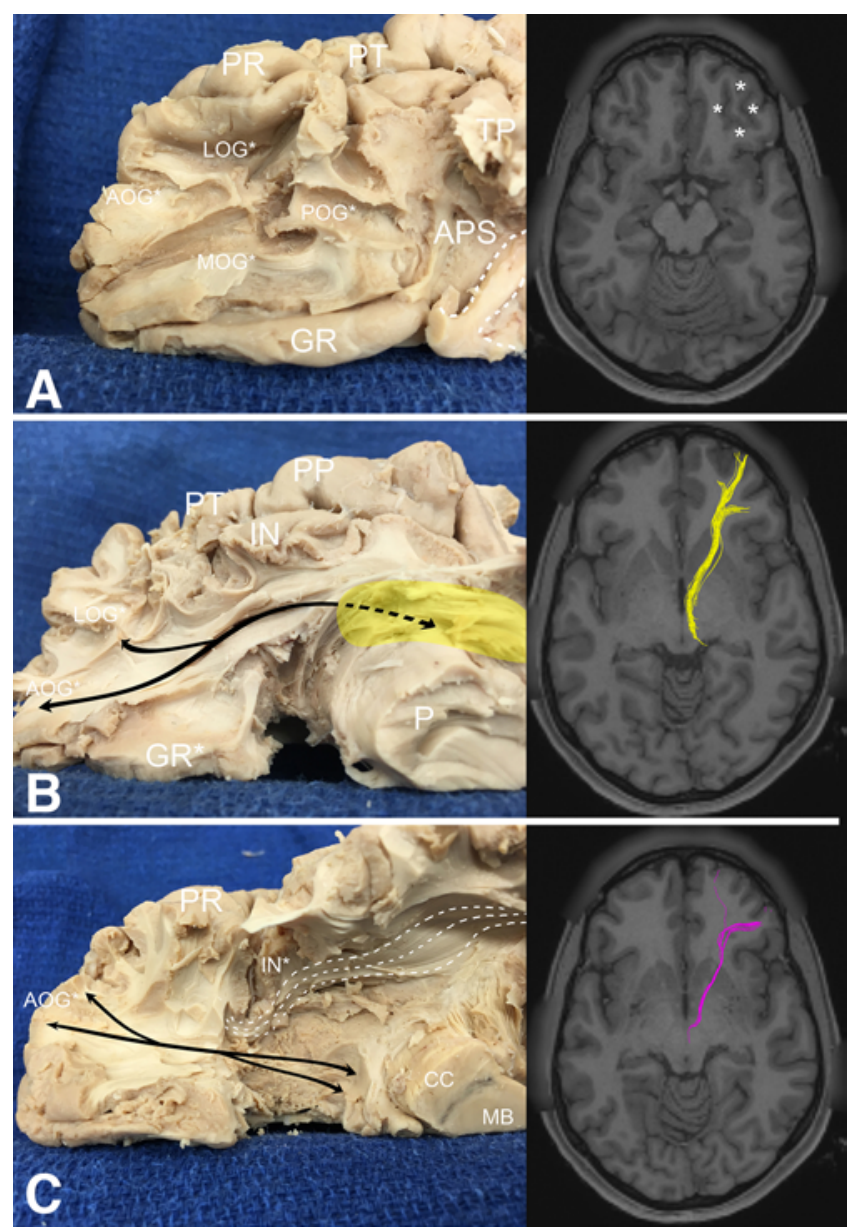

FIG. 2. Orbitofrontal connections to the thalamus and anterior cingulate gyrus. A: Blunt dissection through the anterior portion of the right hemisphere showing the orbital gyri in relation to surrounding structures and an axial T1-weighted MR image with associated asterisks denoting the areas of interest. B: Fiber dissection showing tracts from the orbitofrontal gyri traversing into the superomedial portion of the thalamus, passing lateral to the gyrus rectus (GR), and beneath and lateral to the caudate. Yellow indicates the orbitofrontal connection to the thalamus. Pathway shown by tractography (right) for comparison. C: Fiber dissection revealing path of crossing fibers of the corpus callosum that lie superiormedial to the bundle in B. Pathway illustrated by tractography for comparison. Pink indicates the crossing fibers of the corpus callosum. AOG $=$ anterior orbital gyrus; APS = anterior parolfactory sulcus; $C C=$ crus cerebri; IN = insula; $L O G$ = lateral orbital gyrus; $M B=$ midbrain; $M O G=$ medial orbital gyrus; $P O G=$ posterior orbital gyrus; $P=$ pons; $P P=$ pars opercularis; PR = pars orbitalis.

ital fasciculus (IFOF). Anteriorly, the fibers joining the IFOF originate beneath and lateral to the bundles to the thalamus and brainstem. After passing beneath the limen insula, the bundle connecting to the orbitofrontal gyri divides. The majority of the fibers continue posteriorly to form connections throughout the medial and occipital cortex. Some fibers turn anteriorly with the uncinate fasciculus to connect to anterior portions of the superior and middle temporal gyri.

Of note, fibers traveling more laterally with the IFOF continue to the occipital cortex, while fibers connecting to 

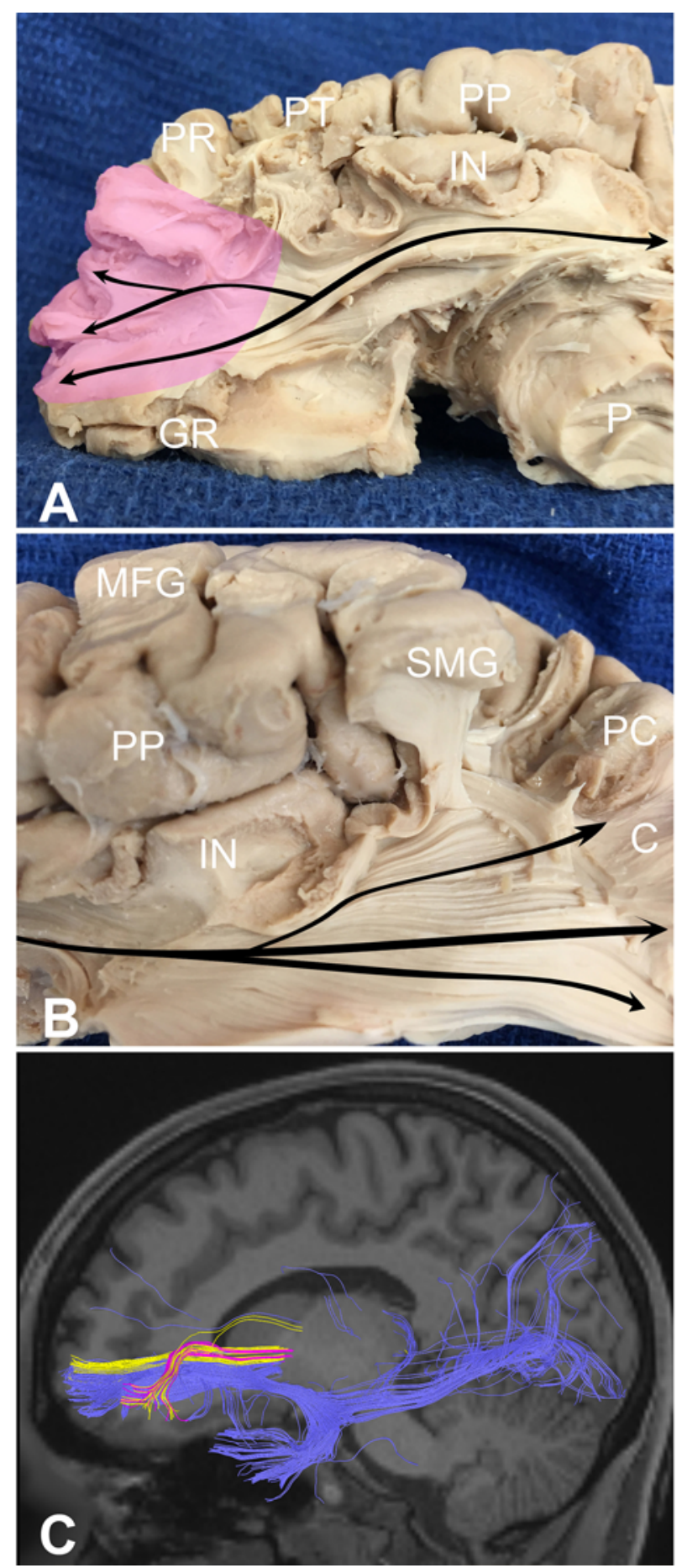

FIG. 3. Orbitofrontal projections to temporal, parietal, and occipital lobes. A: Sagittal dissection through the right hemisphere; fiber dissection revealing connection to the OFG (pink). As the tract passes beneath the limen insula, the bundle connecting to the orbitofrontal gyri divides into superior and inferior tracts as it progresses anteriorly. B: Fiber dissection revealing continuation of posterior fibers to the medial and occipital cortex. Inferior to the insula (IN), the tracts divide into 3 main divisions as they reach the precuneus (PC) and cuneus (C) gyri. C: Tractography demonstrating the orientation of the fibers as they spread posteriorly and anteriorly. As the fibers progress posteriorly, some divide and turn anteriorly to connect to the superior and medial temporal gyri. SMG = supramarginal gyrus. parietal areas course more medially. Following an imaginary line inferiorly from the marginal portion of the cingulate sulcus, which is formed by the junction of the precuneus and the postcentral gyrus, the parietal projections pass medial to the superior longitudinal fasciculus. This is shown in Fig. 3.

\section{Discussion}

In this study, we address connectivity of the OFC between regions important in outcome evaluation and risk assessment. Despite the significance of these networks to daily life, to our knowledge no previous study has described the subcortical anatomy relevant to OFC surgery. The view that functional preservation in cerebral surgery depends on preserving connections between components of functional networks has become broadly accepted. ${ }^{15}$ Using diffusion tractography validated by gross anatomical dissection, we describe the connections of the OFG within the context of surgical anatomy in 10 normal subjects and 10 hemispheres, respectively. We found good concordance between the two methods employed in this study, as have earlier authors..$^{10}$ We focus our discussion primarily on the major tracts connecting to the OFG, as these constitute the bulk of connectivity to other areas of the brain, and are large enough to be clinically actionable. These are the distinct fiber bundles connecting the OFG with the thalamus and brainstem, as well as radiations to the occipital and temporal lobes.

\section{Implications for Cerebral Surgery}

The effect of surgery on tumors involving this network has been subtle, but apparent, in earlier studies. In a 2007 study, Szatkowska et al. described differences in patients undergoing OFC resection, reflecting the likely significance of involvement of either dominant or nondominant sides. They found that patients undergoing dominant-side resection performed worse on the Stroop test, and patients with resection of OFC on either side performed worse on the Trail Making B test. ${ }^{48}$ In a 2011 study, by comparing patients with left-sided lesions to control patients and patients with right-sided OFC lesions, they showed that left-sided OFC plays a crucial role in working memory. ${ }^{49}$ However, based on their results, right-sided OFC may still modulate some aspects of guiding behavior.

Additionally, patients with postsurgical, unilateral lesions to the OFC have shown deficits in facial expression identification, social behavior, and emotional state..$^{6,23}$ Nonsurgical lesions to the same area have led to similar results, with bilateral lesions being more dramatic.6,19,26 These changes are notably occult, given the minimal changes to memory, motor function, language, and executive function. ${ }^{1}$ Fitting with our understanding of OFG and emotion, patients with tumors in proximity to OFG have been observed to perform significantly worse on emotional response testing. ${ }^{26}$ However, most likely due to lesser white matter tract damage, radiation does not seem to affect this network. ${ }^{7}$ Additionally, fMRI studies have shown recruitment of different cortical areas during executive action in some tumor patients, suggesting that the OFG, similar to motor and speech areas, ${ }^{27,44}$ has the ability to reorganize. ${ }^{40}$ 


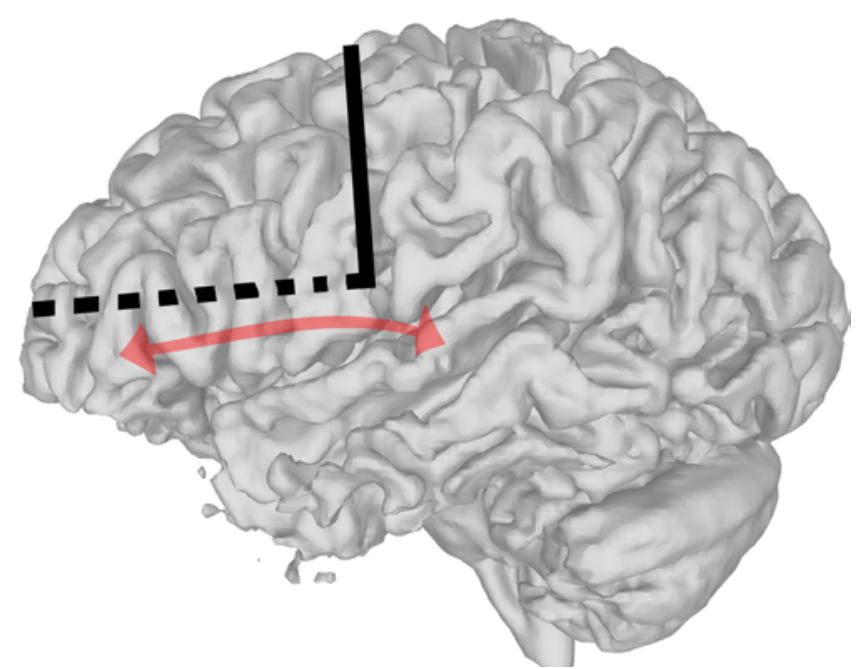

FIG. 4. Schematic representing a technique for preservation of orbitofrontal connectivity (red solid line). Tractography incorporated into image guidance and awake brain mapping are often employed during the posterior disconnection (solid black line) of a frontal lobectomy to preserve language functioning. Similarly, tractography with intraoperative attention testing may aid in preserving functionality of the orbitofrontal gyri during resection of the inferior aspect of the tumor (dashed black line).

We suggest that while OFC connectivity cannot be saved in every case, that when oncologically feasible, it should be saved because 1) it is subtly eloquent brain and OFC resection can impair function in some people, and 2) there are limited direct connections between the OFC and the rest of the brain. In presenting the connections of the OFC in their entirety, along with relevant surgical anatomy, we offer the location and nature of tracts that may be affected by frontal tumor surgery. Earlier studies have focused on functioning of the OFG or on surface anatomy, ${ }^{43}$ limiting their direct utility in tumor surgery. With a working understanding of the subcortical anatomy and the location of these tracts relative to the tumor, surgeons can plan operative approaches that further minimize risk to the patient.

For instance, in a frontal lobectomy, the primary focus is on preserving anatomy associated with language, such as Broca's area and the superior longitudinal fasciculus. ${ }^{9}$ These can be disrupted during the posterior disconnection of the frontal lobe. However, with awake mapping and careful operative planning with image guidance, postoperative aphasia is a rare complication. ${ }^{8}$ Yet, standard intraoperative testing for frontal lobe function is not yet established. Thus, we propose limiting the inferior aspect of this cut when possible, as it may be useful in preserving salient connections of the orbitofrontal areas to the midbrain and thalamic nuclei (Fig. 4). This can be achieved using standard image-guidance platforms (Fig. 5).

One of the major challenges in highlighting the clinical implications of operating in this region has been limited availability of reproducible tests for executive function. ${ }^{11}$ Contemporary neurosurgical literature has placed a heavy emphasis on outcomes with respect to survival and global functioning, without examining detailed cogni-
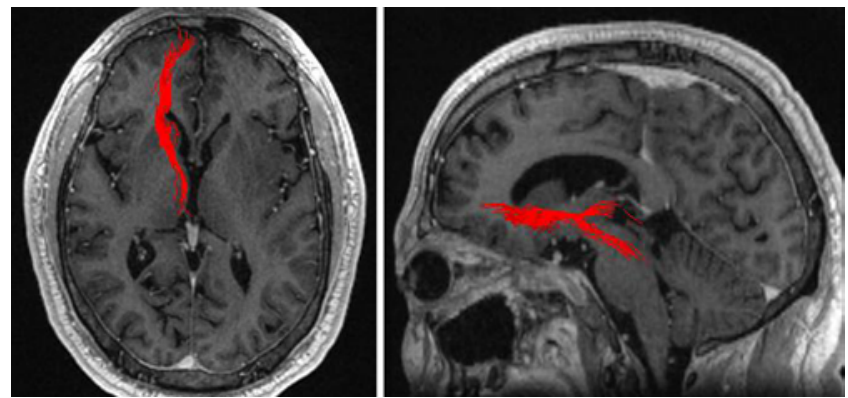

FIG. 5. Use of a standard image-guidance platform for the identification of major OFG connections. Screenshots from an MRI navigation map with tractography overlay (red) in the axial (left) and sagittal (right) planes demonstrate how the anatomy described in this study may be used for surgical planning. These tracts were visualized using a StealthViz planning station (Medtronic) by a nonexpert in less than 5 minutes. Major fiber bundles to the thalamus and midbrain are shown.

tive status pre- and postoperatively. This currently limits conclusions that can be drawn about anatomo-functional correlates within the frontal lobe. Future work in this area may indeed be focused on improved methodologies for systematically examining patients with orbitofrontal pathology with standardized neuropsychological testing. An improved understanding from a functional standpoint will also better inform decisions regarding the limits of resection.

\section{Relation of Gross Dissection to Tractography}

Unsurprisingly, gross dissection did not allow visualization of every individual tract seen with tractography. Generalized q-sampling (GQI) allowed us to perform tractography in voxels that contained crossing fibers, ${ }^{58}$ which was particularly useful in proximity to the brainstem. Where gross anatomical dissections allow for qualitative understanding of tract orientation, tractography allowed us to quantify tract volume to better understand laterality. In freeze-fractured brains, major tracts were clearly evident. As such, prior knowledge would have influenced DTI results to a much greater extent than findings in anatomical dissection. The results of the cadaveric study are primarily limited by the technique, which is aimed at larger, macroscopic anatomy.

The general morphology of the major tracts was constant across specimens: medial fibers of the OFG connect to the thalamus, with the fibers connecting to the brainstem coursing more laterally. Lateral and inferior to these were the radiations traveling with the IFOF, which connected to the posterolateral portion of the OFG.

\section{Orbitofrontal Cortex and Sensory Processing}

All functions attributed to the medial and lateral OFG require sensory input, and various models of interaction have been proposed. Previously, these projections have been noted in nonhuman primate ${ }^{5,12}$ and DTI ${ }^{25}$ studies. The parietal and occipital radiations noted in our tractography present a direct pathway for information from spatial and visual association cortices to reach the OFG. Given the role of the OFC in reward pathways, ${ }^{16,21,30}$ connections be- 
tween OFG and visual association cortex likely reinforce the positive visual identification of an object. ${ }^{33}$ That is to say, during the act of visually searching for an object, the OFC is important in deciding whether the object visualized is the target. ${ }^{25,31,33,36}$ This process helps explain activation of reward pathways associated with visualizing currency, which is known to play a role in gambling and other risk-based decisions. . $^{14,31,36}$ Consequently, patients affected by tumors of the OFC can experience behavioral changes resulting from changes in perception of risk. One recent study showed that meningioma patients with tumors involving the OFC had poor postoperative scores on the Iowa Gambling Task. ${ }^{1}$ Interestingly, these patients had no overall change in attention, perception, memory, language, or executive function as measured by a standardized neuropsychological evaluation.

Involvement of the OFG in reward pathways plays a role in the learning of spatial relationships through parietal connections..$^{13,45}$ These connections can explain why the perception of reward is proportional to an individual's distance from a target. ${ }^{32}$ These relationships fit with accepted views of the OFC as a center for evaluation control. ${ }^{46}$ However, this functional relationship is subtle, and it is difficult to appreciate clinically, as lesions of the parietal lobe can have countless manifestations.

\section{Orbitofrontal Cortex and Emotion}

We elected to study the laterality of the fiber bundle connecting the OFG to the thalamus, as this connection seems to be the most critical in executive functioning. Proximal connections between the thalamus and OFC and the anterior cingulate and OFC have been the subject of many animal studies. ${ }^{2,21,24}$ These connections have been extensively linked to cognition, memory, and emotion. ${ }^{5}$ Projections from the mediodorsal thalamus in particular have an established role in emotion and reward linked to decision making. ${ }^{39}$ These tracts have been studied in a number of psychological processes. . $^{51,53,54}$ Well-known examples include a link between treating epilepsy of the OFC and reversible antisocial behavior. ${ }^{51}$ There is also a link between schizophrenia and tract abnormalities connecting the OFC to the anterior cingulate cortex. ${ }^{38,46,53}$ Many behavioral/emotional disturbances make sense within the context of the OFC as important for evaluation control. ${ }^{46}$

As a whole, this network has been used in the treatment of several conditions. For instance, disconnecting tracts from the thalamus to the OFC has been successful in treating obsessive-compulsive disorder (OCD) with subcaudate tractotomy. ${ }^{22,57}$ Deep brain stimulation has also been successful in treating OCD ${ }^{41}$ likely by acting on connections of the OFC to the thalamus in the ventral capsule/ventral striatum region. ${ }^{34}$

\section{Conclusions}

The OFG is an important center for processing visual, spatial, and emotional information. Subtle differences in executive functioning following surgery for frontal lobe tumors may be better understood in the context of the fiber-bundle anatomy highlighted by this study.

\section{References}

1. Abel TJ, Manzel K, Bruss J, Belfi AM, Howard MA III, Tranel D: The cognitive and behavioral effects of meningioma lesions involving the ventromedial prefrontal cortex. J Neurosurg 124:1568-1577, 2016

2. Alcaraz F, Marchand AR, Vidal E, Guillou A, Faugère A, Coutureau E, et al: flexible use of predictive cues beyond the orbitofrontal cortex: role of the submedius thalamic nucleus. J Neurosci 35:13183-13193, 2015

3. Alvarez JA, Emory E: Executive function and the frontal lobes: a meta-analytic review. Neuropsychol Rev 16:17-42, 2006

4. Ardekani BA, Tabesh A, Sevy S, Robinson DG, Bilder RM, Szeszko PR: Diffusion tensor imaging reliably differentiates patients with schizophrenia from healthy volunteers. Hum Brain Mapp 32:1-9, 2011

5. Barbas H: Connections underlying the synthesis of cognition, memory, and emotion in primate prefrontal cortices. Brain Res Bull 52:319-330, 2000

6. Bramham J, Morris RG, Hornak J, Bullock P, Polkey CE: Social and emotional functioning following bilateral and unilateral neurosurgical prefrontal cortex lesions. J Neuropsychol 3:125-143, 2009

7. Brummelman P, Sattler MG, Meiners LC, Elderson MF, Dullaart RP, van den Berg G, et al: Cognitive performance after postoperative pituitary radiotherapy: a dosimetric study of the hippocampus and the prefrontal cortex. Eur J Endocrinol 166:171-179, 2012

8. Burks JD, Bonney PA, Conner AK, Glenn CA, Briggs RG, Battiste JD, et al: A method for safely resecting anterior butterfly gliomas: the surgical anatomy of the default mode network and the relevance of its preservation. J Neurosurg [epub ahead of print September 16, 2016. DOI: 10.3171/2016.5.JNS153006]

9. Burks JD, Conner AK, Bonney PA, Glenn CA, Smitherman $\mathrm{AD}$, Ghafil CA, et al: Frontal keyhole craniotomy for resection of low- and high-grade gliomas. Neurosurgery [epub ahead of print], 2017

10. Catani M, Dell'Acqua F, Vergani F, Malik F, Hodge H, Roy P, et al: Short frontal lobe connections of the human brain. Cortex 48:273-291, 2012

11. Chan RC, Shum D, Toulopoulou T, Chen EY: Assessment of executive functions: review of instruments and identification of critical issues. Arch Clin Neuropsychol 23:201-216, 2008

12. Chavis DA, Pandya DN: Further observations on corticofrontal connections in the rhesus monkey. Brain Res 117:369-386, 1976

13. Colby CL, Goldberg ME: Space and attention in parietal cortex. Annu Rev Neurosci 22:319-349, 1999

14. Della Libera C, Chelazzi L: Visual selective attention and the effects of monetary rewards. Psychol Sci 17:222-227, 2006

15. Duffau H: The challenge to remove diffuse low-grade gliomas while preserving brain functions. Acta Neurochir (Wien) 154:569-574, 2012

16. Elliott R, Dolan RJ, Frith CD: Dissociable functions in the medial and lateral orbitofrontal cortex: evidence from human neuroimaging studies. Cereb Cortex 10:308-317, 2000

17. Francis S, Rolls ET, Bowtell R, McGlone F, O'Doherty J, Browning A, et al: The representation of pleasant touch in the brain and its relationship with taste and olfactory areas. Neuroreport 10:453-459, 1999

18. Funahashi S: [The contribution of the orbitofrontal cortex to the preference for visual stimuli.] Brain Nerve 67:711-722, 2015 (Jpn)

19. Goodkind MS, Sollberger M, Gyurak A, Rosen HJ, Rankin KP, Miller B, et al: Tracking emotional valence: the role of the orbitofrontal cortex. Hum Brain Mapp 33:753-762, 2012

20. Gottfried JA, O’Doherty J, Dolan RJ: Encoding predictive 
reward value in human amygdala and orbitofrontal cortex. Science 301:1104-1107, 2003

21. Groenewegen HJ: Organization of the afferent connections of the mediodorsal thalamic nucleus in the rat, related to the mediodorsal-prefrontal topography. Neuroscience 24:379431, 1988

22. Hartmann CJ, Lujan JL, Chaturvedi A, Goodman WK, Okun MS, McIntyre CC, et al: Tractography activation patterns in dorsolateral prefrontal cortex suggest better clinical responses in OCD DBS. Front Neurosci 9:519, 2016

23. Hornak J, Bramham J, Rolls ET, Morris RG, O’Doherty J, Bullock PR, et al: Changes in emotion after circumscribed surgical lesions of the orbitofrontal and cingulate cortices. Brain 126:1691-1712, 2003

24. Jackson SA, Horst NK, Pears A, Robbins TW, Roberts AC: Role of the perigenual anterior cingulate and orbitofrontal cortex in contingency learning in the marmoset. Cereb Cortex 26:3273-3284, 2016

25. Jarbo K, Verstynen TD: Converging structural and functional connectivity of orbitofrontal, dorsolateral prefrontal, and posterior parietal cortex in the human striatum. J Neurosci 35:3865-3878, 2015

26. Jenkins LM, Andrewes DG, Nicholas CL, Drummond KJ, Moffat BA, Phal P, et al: Social cognition in patients following surgery to the prefrontal cortex. Psychiatry Res 224:192-203, 2014

27. Jungblut M, Huber W, Mais C, Schnitker R: Paving the way for speech: voice-training-induced plasticity in chronic aphasia and apraxia of speech - three single cases. Neural Plast 2014:841982, 2014

28. Kawamura M, Miller MW, Ichikawa H, Ishihara K, Sugimoto A: Brodmann area 12: an historical puzzle relevant to FTLD. Neurology 76:1596-1599, 2011

29. Koutsarnakis C, Liakos F, Kalyvas AV, Sakas DE, Stranjalis G: A laboratory manual for stepwise cerebral white matter fiber dissection. World Neurosurg 84:483-493, 2015

30. Kringelbach ML: The human orbitofrontal cortex: linking reward to hedonic experience. Nat Rev Neurosci 6:691-702, 2005

31. Kristjánsson A, Sigurjónsdóttir O, Driver J: Fortune and reversals of fortune in visual search: Reward contingencies for pop-out targets affect search efficiency and target repetition effects. Atten Percept Psychophys 72:1229-1236, 2010

32. Lee J, Shomstein S: The differential effects of reward on space- and object-based attentional allocation. J Neurosci 33:10625-10633, 2013

33. Lee J, Shomstein S: Reward-based transfer from bottom-up to top-down search tasks. Psychol Sci 25:466-475, 2014

34. Makris N, Rathi Y, Mouradian P, Bonmassar G, Papadimitriou G, Ing WI, et al: Variability and anatomical specificity of the orbitofrontothalamic fibers of passage in the ventral capsule/ventral striatum (VC/VS): precision care for patient-specific tractography-guided targeting of deep brain stimulation (DBS) in obsessive compulsive disorder (OCD). Brain Imaging Behav 10:1054-1067, 2016

35. Martino J, De Witt Hamer PC, Vergani F, Brogna C, de Lucas EM, Vázquez-Barquero A, et al: Cortex-sparing fiber dissection: an improved method for the study of white matter anatomy in the human brain. J Anat 219:531-541, 2011

36. Navalpakkam V, Koch C, Rangel A, Perona P: Optimal reward harvesting in complex perceptual environments. Proc Natl Acad Sci US A 107:5232-5237, 2010

37. Nelson JK, Reuter-Lorenz PA, Persson J, Sylvester CY, Jonides J: Mapping interference resolution across task domains: a shared control process in left inferior frontal gyrus. Brain Res 1256:92-100, 2009

38. Ohtani T, Bouix S, Hosokawa T, Saito Y, Eckbo R, Ballinger $\mathrm{T}$, et al: Abnormalities in white matter connections between orbitofrontal cortex and anterior cingulate cortex and their associations with negative symptoms in schizophrenia: a DTI study. Schizophr Res 157:190-197, 2014

39. Oyoshi T, Nishijo H, Asakura T, Takamura Y, Ono T: Emotional and behavioral correlates of mediodorsal thalamic neurons during associative learning in rats. J Neurosci 16:5812-5829, 1996

40. Ozyurt J, Lorenzen A, Gebhardt U, Warmuth-Metz M, Müller HL, Thiel CM: Remote effects of hypothalamic lesions in the prefrontal cortex of craniopharygioma patients. Neurobiol Learn Mem 111:71-80, 2014

41. Pepper J, Hariz M, Zrinzo L: Deep brain stimulation versus anterior capsulotomy for obsessive-compulsive disorder: a review of the literature. J Neurosurg 122:1028-1037, 2015

42. Ribas GC: The cerebral sulci and gyri. Neurosurg Focus 28(2):E2, 2010

43. Rodrigues TP, Rodrigues MA, de Araújo Paz D, Silva da Costa MD, Centeno RS, Chaddad Neto FE, et al: Orbitofrontal sulcal and gyrus pattern in human: an anatomical study. Arq Neuropsiquiatr 73:431-435, 2015

44. Sengupta R, Nasir SM: The predictive roles of neural oscillations in speech motor adaptability. J Neurophysiol 115:2519-2528, 2016

45. Shomstein S, Kravitz DJ, Behrmann M: Attentional control: temporal relationships within the fronto-parietal network. Neuropsychologia 50:1202-1210, 2012

46. Solbakk AK, Løvstad M: Effects of focal prefrontal cortex lesions on electrophysiological indices of executive attention and action control. Scand J Psychol 55:233-243, 2014

47. Stroop JR: Studies of interference in serial verbal reactions. J Exp Psychol 18:643-662, 1935

48. Szatkowska I, Szymańska O, Bojarski P, Grabowska A: Cognitive inhibition in patients with medial orbitofrontal damage. Exp Brain Res 181:109-115, 2007

49. Szatkowska I, Szymańska O, Marchewka A, Soluch P, Rymarczyk K: Dissociable contributions of the left and right posterior medial orbitofrontal cortex in motivational control of goal-directed behavior. Neurobiol Learn Mem 96:385391,2011

50. Thiebaut de Schotten M, Dell'Acqua F, Forkel SJ, Simmons A, Vergani F, Murphy DG, et al: A lateralized brain network for visuospatial attention. Nat Neurosci 14:1245-1246, 2011

51. Trebuchon A, Bartolomei F, McGonigal A, Laguitton V, Chauvel P: Reversible antisocial behavior in ventromedial prefrontal lobe epilepsy. Epilepsy Behav 29:367-373, 2013

52. Wager M, Du Boisgueheneuc F, Pluchon C, Bouyer C, Stal $\mathrm{V}$, Bataille B, et al: Intraoperative monitoring of an aspect of executive functions: administration of the Stroop test in 9 adult patients during awake surgery for resection of frontal glioma. Neurosurgery 72 (2 Suppl Operative):ons169ons181, 2013

53. Wagner G, De la Cruz F, Schachtzabel C, Güllmar D, Schultz CC, Schlösser RG, et al: Structural and functional dysconnectivity of the fronto-thalamic system in schizophrenia: a DCM-DTI study. Cortex 66:35-45, 2015

54. Wolf RC, Philippi CL, Motzkin JC, Baskaya MK, Koenigs M: Ventromedial prefrontal cortex mediates visual attention during facial emotion recognition. Brain 137:1772-1780, 2014

55. Wu JS, Zhou LF, Tang WJ, Mao Y, Hu J, Song YY, et al: Clinical evaluation and follow-up outcome of diffusion tensor imaging-based functional neuronavigation: a prospective, controlled study in patients with gliomas involving pyramidal tracts. Neurosurgery 61:935-949, 2007

56. Yan C, Su L, Wang Y, Xu T, Yin DZ, Fan MX, et al: Multivariate neural representations of value during reward anticipation and consummation in the human orbitofrontal cortex. Sci Rep 6:29079, 2016

57. Yang JC, Papadimitriou G, Eckbo R, Yeterian EH, Liang 
L, Dougherty DD, et al: Multi-tensor investigation of orbitofrontal cortex tracts affected in subcaudate tractotomy. Brain Imaging Behav 9:342-352, 2015

58. Yeh FC, Wedeen VJ, Tseng WY: Generalized q-sampling imaging. IEEE Trans Med Imaging 29:1626-1635, 2010

\section{Disclosures}

The authors report no conflict of interest concerning the materials or methods used in this study or the findings specified in this paper.

\section{Author Contributions}

Conception and design: Sughrue, Burks, Bonney. Acquisition of data: Burks, Conner, O'Donoghue, Wu. Analysis and interpretation of data: Burks, Conner, O'Donoghue, Wu. Drafting the article: Sughrue, Burks, Conner, Bonney, Glenn, Boettcher. Critically revising the article: Sughrue, Burks, Glenn, Baker, Boettcher, Briggs. Reviewed submitted version of manuscript: Baker. Administrative/technical/material support: Sughrue, Baker.

\section{Correspondence}

Michael E. Sughrue, Department of Neurological Surgery, University of Oklahoma Health Sciences Center, 1000 N Lincoln Blvd., Ste. 4000, Oklahoma City, OK 73104. email: michael-sughrue@ ouhsc.edu. 